\title{
Clinical-Radiomic Analysis for Pretreatment Prediction of Objective Response to First Transarterial Chemoembolization in Hepatocellular Carcinoma
}

\author{
Mingyu Chen ${ }^{a, b, c}$ Jiasheng Cao ${ }^{a}$ Jiahao Hu ${ }^{a}$ Win Topatana ${ }^{c}$ Shijie $\mathrm{Li}^{\mathrm{a}}$ \\ Sarun Juengpanich ${ }^{c}$ Jian Lin $^{d}$ Chenhao Tong ${ }^{\mathrm{e}}$ Jiliang Shen $^{\mathrm{a}}$ Bin Zhang $^{\mathrm{a}}$ \\ Jennifer Wu ${ }^{f}$ Christine Pochag ${ }^{g}$ Masatoshi Kudo ${ }^{\mathrm{h}} \quad$ Amedeo Amedei $^{\mathrm{i}}$ \\ Franco Trevisani ${ }^{j}$ Pil Soo Sung ${ }^{k}$ Victor M. Zaydfudim' Tatsuo Kandam \\ Xiujun Cai a, b, n AME Hepatocellular Carcinoma Collaborative Group

\begin{abstract}
aDepartment of General Surgery, Sir Run-Run Shaw Hospital, Zhejiang University, Hangzhou, China; ${ }^{b}$ Engineering Research Center of Cognitive Healthcare of Zhejiang Province, Hangzhou, China; 'Zhejiang University School of Medicine, Hangzhou, China; ' General Surgery, Longyou People's Hospital, Quzhou, China; 'General Surgery, Shaoxing People's Hospital, Shaoxing, China; ${ }^{P}$ Perlmutter Cancer Center, NYU Langone Health, New York, NY, USA; ${ }^{9}$ Avera McKennnan Hospital and University Medical Center, Sanford School of Medicine, University of South Dakota, Sioux Falls, SD, USA; hepartment of Gastroenterology and Hepatology, Kindai University School of Medicine, Osaka, Japan; 'Department of Experimental and Clinical Medicine, University of Florence, Florence, Italy; 'Department of Medical and Surgical Sciences, Semeiotica Medica, Alma Mater Studiorum, University of Bologna,

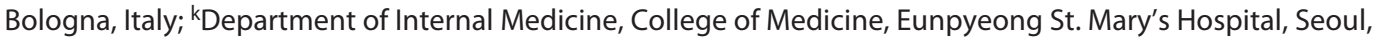
Republic of Korea; 'Department of Surgery, Section of Hepatobiliary and Pancreatic Surgery, University of Virginia, Charlottesville, VA, USA; 'mivision of Gastroenterology and Hepatology, Department of Medicine, Nihon University School of Medicine, Tokyo, Japan; " Zhejiang Research and Development Engineering Laboratory of Minimally
\end{abstract} \\ Invasive Technology and Equipment, Hangzhou, China
}

\section{Keywords}

Hepatocellular carcinoma - Transarterial

chemoembolization - Treatment response $\cdot$ Radiomics

\begin{abstract}
Background: The preoperative selection of patients with intermediate-stage hepatocellular carcinoma (HCC) who are likely to have an objective response to first transarterial chemoembolization (TACE) remains challenging. Objective: To develop and validate a clinical-radiomic model (CR model) for preoperatively predicting treatment response to first
\end{abstract}

karger@karger.com www.karger.com/lic

Karger"

BOPEN ACCESS
(C) 2021 The Author(s)

Published by S. Karger AG, Basel

This article is licensed under the Creative Commons AttributionNonCommercial-NoDerivatives 4.0 International License (CC BYNC-ND) (http://www.karger.com/Services/OpenAccessLicense) Usage and distribution for commercial purposes as well as any distribution of modified material requires written permission.
TACE in patients with intermediate-stage HCC. Methods: A total of 595 patients with intermediate-stage HCC were included in this retrospective study. A tumoral and peritumoral $(10 \mathrm{~mm})$ radiomic signature (TPR-signature) was constructed based on 3,404 radiomic features from 4 regions of interest. A predictive CR model based on TPR-signature and clinical factors was developed using multivariate logistic regression. Calibration curves and area under the receiver operating characteristic curves (AUCs) were used to evaluate the model's performance. Results: The final CR model consisted of 5 independent predictors, including TPR-signature $(p<0.001)$, AFP $(p=0.004)$, Barcelona Clinic Liver Cancer Sys- 
tem Stage B (BCLC B) subclassification ( $p=0.01$ ), tumor location $(p=0.039)$, and arterial hyperenhancement $(p=0.050)$. The internal and external validation results demonstrated the high-performance level of this model, with internal and external AUCs of 0.94 and 0.90 , respectively. In addition, the predicted objective response via the CR model was associated with improved survival in the external validation cohort (hazard ratio: 2.43 ; 95\% confidence interval: $1.60-3.69 ; p<$ 0.001 ). The predicted treatment response also allowed for significant discrimination between the Kaplan-Meier curves of each BCLC B subclassification. Conclusions: The CR model had an excellent performance in predicting the first TACE response in patients with intermediate-stage HCC and could provide a robust predictive tool to assist with the selection of patients for TACE.

(C) 2021 The Author(s)

Published by S. Karger AG, Basel

\section{Introduction}

Hepatocellular carcinoma (HCC) is the fifth leading cause of cancer mortality [1] and the most common cause of liver-related death [2], with $>850,000$ associated deaths reported annually worldwide [3,4]. Patients with early HCC are candidates for curative therapies, such as transplantation, resection, and ablation, and have an overall 5 -year survival rate reaching to $70-80 \%[5,6]$. However, many patients with HCC are diagnosed at the intermediate-stage Barcelona Clinic Liver Cancer staging classification B (BCLC B), with significantly decreased survival compared to patients with early-stage, BCLC A HCC [7]. According to the European and American guidelines for the management of HCC, transarterial chemoembolization (TACE) is recommended as the first-line therapy for patients with intermediate-stage HCC [8-10]. HCC patients with an objective response to first TACE enjoy a survival benefit [11], but nearly half of the HCC patients are nonresponsive to first TACE and have a poor prognosis [12]. For HCC patients who show no response to TACE, timely conversion to sorafenib or lenvatinib can prevent further liver dysfunction and prolong overall survival (OS), particularly if targeted kinase inhibitor therapy can be initiated before the HCC progresses to the advanced stage [13-15].

Several clinical factors are beneficial for preoperatively selecting patients who are likely to have an objective response to TACE $[16,17]$. The presence of fewer tumors, tumor size $<5 \mathrm{~cm}$, and a higher proportion of arterial enhanced tumors have been associated with a better treatment response rate [18]. On the contrary, hypovascular tumors and those located in segment I or IV have been associated with poorer treatment response $[18,19]$. Several pre-TACE prediction models that were developed to estimate treatment response to TACE showed promising initial results [20-25]. However, those models did not incorporate tumor biology, a factor which might lead to a decrease in performance [26].

Recent advances in radiomics have provided a robust method for extracting radiological features beyond what is possible with the human eye, with the ability to assess tumor biological characteristics and oncologic prognoses [27]. The appropriate integration of radiomic features and clinical factors can improve accuracy in complex clinical decision-making [28].

Therefore, we aimed to establish a clinical-radiomic model (CR model) that integrated patient-specific information and radiomic features extracted from contrastenhanced computed tomography (CECT) (1) to accurately predict response to first TACE and (2) to assess its significance for OS in patients with intermediate-stage HCC. This model was further validated with an independent external cohort.

\section{Materials and Methods}

\section{Study Population}

A total of 595 HCC patients between January 2010 and December 2014 were included in this study according to the following inclusion criteria: (1) radiologically and/or pathologically confirmed HCC; (2) BCLC B; (3) CECT performed within 1 month prior to the initial TACE treatment; and (4) the availability of CECT within 2 months of the first TACE. The exclusion criteria were (1) patients under 18 years old; (2) patients who had received other treatments, such as transplantation, resection, ablation, or targeted drugs, before the initial TACE treatment; (3) the presence of other cancers; or (4) a lack of necessary clinical information on the patient's demographic characteristics, laboratory examinations, tumor characteristics, treatment details, and follow-up. A total of 473 patients from Sir Run-Run Shaw Hospital, Zhejiang University, were identified as the primary cohort, which was randomly divided into a training cohort $(N=355)$ and an internal validation cohort $(N=118)$, based on a random split-sample $(3: 1)$ approach (Fig. 1). The external validation cohort comprised 122 patients who fulfilled the selection criteria from 2 other medical centers (Shaoxing People's Hospital and Longyou People's Hospital).

A standardized data form was created to collect all relevant clinical information. The form included 27 items which were categorized into the 4 following groups: (1) the demographics and clinical characteristics of the patients (including age, gender, hypertension, diabetes, cirrhosis, hepatitis, and antiviral treatment); (2) laboratory variables including alpha-fetoprotein (AFP), alanine aminotransferase (ALT), aspartate aminotransferase (AST), albumin (ALB), total bilirubin (TB), prothrombin time (PT), albu- 


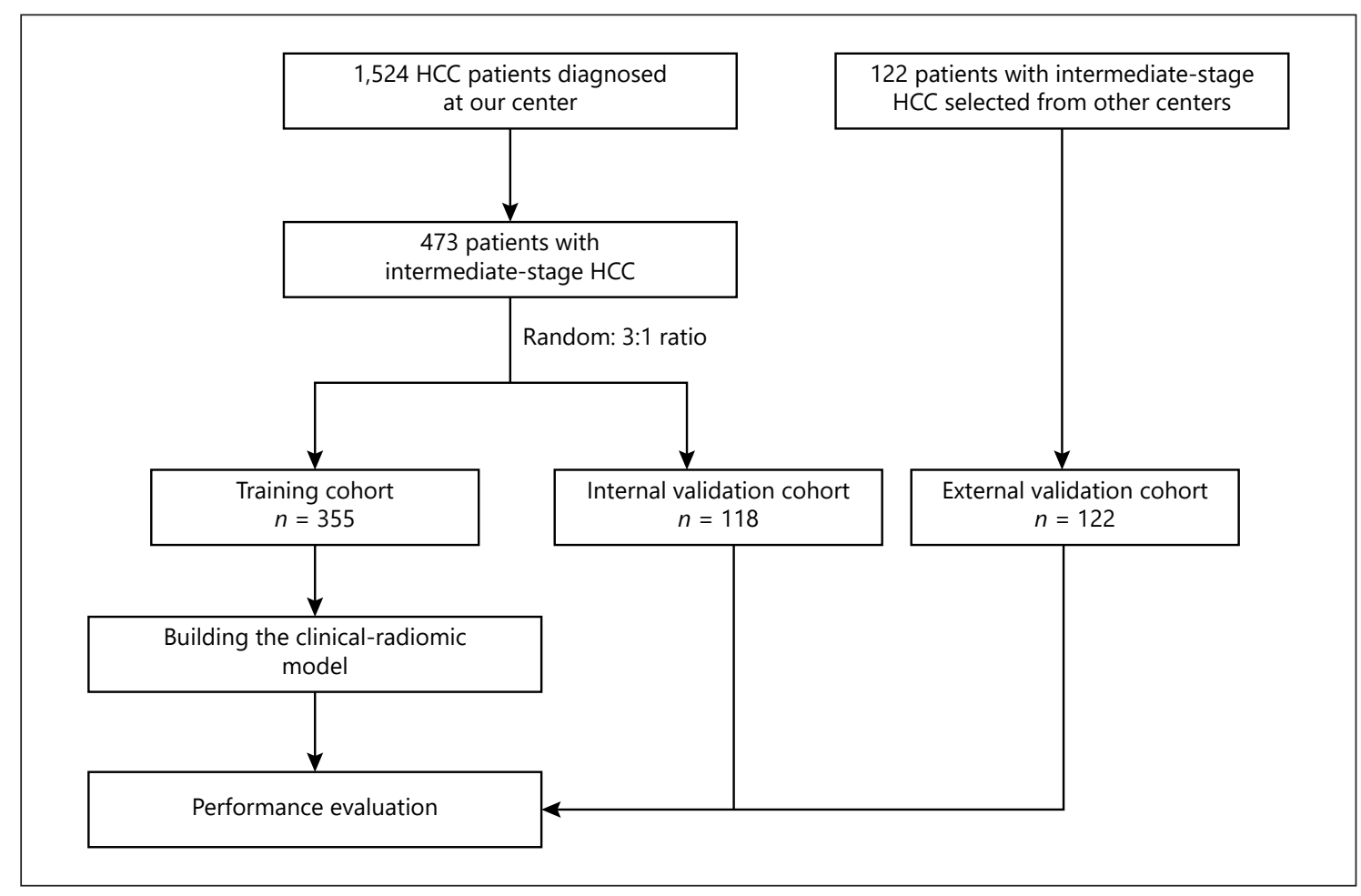

Fig. 1. Flowchart of patients and study design. HCC, hepatocellular carcinoma; CR, clinical radiomic.

min-bilirubin (ALBI) grade $[29,30]$, Child-Pugh class, and BCLC B subclassification (B1-B4) [31]; (3) tumor characteristics, such as number, size (of the largest lesion in multinodular HCC), location (targeted HCC and nontargeted lesions), and arterial hyperenhancement status on CECT scan; and (4) details of treatment and follow-up.

Treatment Response and Follow-Up

Treatment response to initial TACE was evaluated on a pertarget nodule and a per-patient basis on the follow-up CECT imaging using the modified Response Evaluation Criteria in Solid Tumors (mRECIST) $[13,15]$. Notably, treatment response to initial TACE was divided into objective response (complete or partial response) and nonresponsive (stable disease or progressive disease). Treatment response was independently evaluated by 2 faculty radiologists with $>6$ years of experience, and conflicting results were mitigated by a third radiologist with over 10 years of experience.

Follow-up visits were performed with laboratory variables including AFP, ALT, AST, ALB, TB, and PT and abdominal CECT recorded every 2-3 months. Subsequent treatments, such as repeated TACE, thermal ablation, sorafenib, or supportive care (TACE was sometimes used in combination with ablation, sorafenib, or supportive care), were also recorded.

Segmentation of ROIs and Acquisition of Radiomic Features

All preoperative CECT images in our study met the criteria delineated by the American Association for the Study of Liver Disease (AASLD) guidelines [9]. First, all images were initially col- lected, prejudged, and independently scored by 2 radiologists based on 10 primary imaging features, which were recommended by Xu et al. [32], Banerjee et al. [33], and Wang et al. [34]. The details of the CT imaging techniques and prejudged criteria are summarized and described in online suppl. Text 1 (for all online suppl. material, see www.karger.com/doi/10.1159/000512028). Notably, for multinodular HCC, only the targeted lesions (the largest nodules) with abundant vascularity that were easy to analyze with radiomics were selected for further analysis, while other nontargeted lesions were estimated using radiological information. Interobserver discrepancies between scores for the same image, including images of the targeted lesions, were reviewed by 3 radiologists to reach a consensus before the region of interest (ROI) segmentation was performed.

The ROIs were drawn at each targeted lesion by 2 radiologists using open-source 3D slicer V4.10.2 (https://www.slicer.org/) software, which provided a powerful function of semiautomatic segmentation. The tumoral ROI was considered to the entire tumor volume (main part), and the peritumoral ROI was considered to be the peritumoral tissues (expanded rim part), which were reconstructed with a dilation algorithm by 5,10 , and $20 \mathrm{~mm}$, respectively. If the longest distance from the liver surface to the tumor surface was shorter than the dilation distance, the regions with the longest distance for superficial lesions were identified as peritumoral ROIs. According to the performances in the training and testing sets, the dilation distance in the model with the best performance was identified for drawing further peritumoral ROIs (expanded rim part). Then, the tumoral ROI (main part) and peritumoral ROI (expanded rim part) were drawn at the hepatic arterial
40

Liver Cancer 2021;10:38-51

DOI: $10.1159 / 000512028$
Chen et al. 

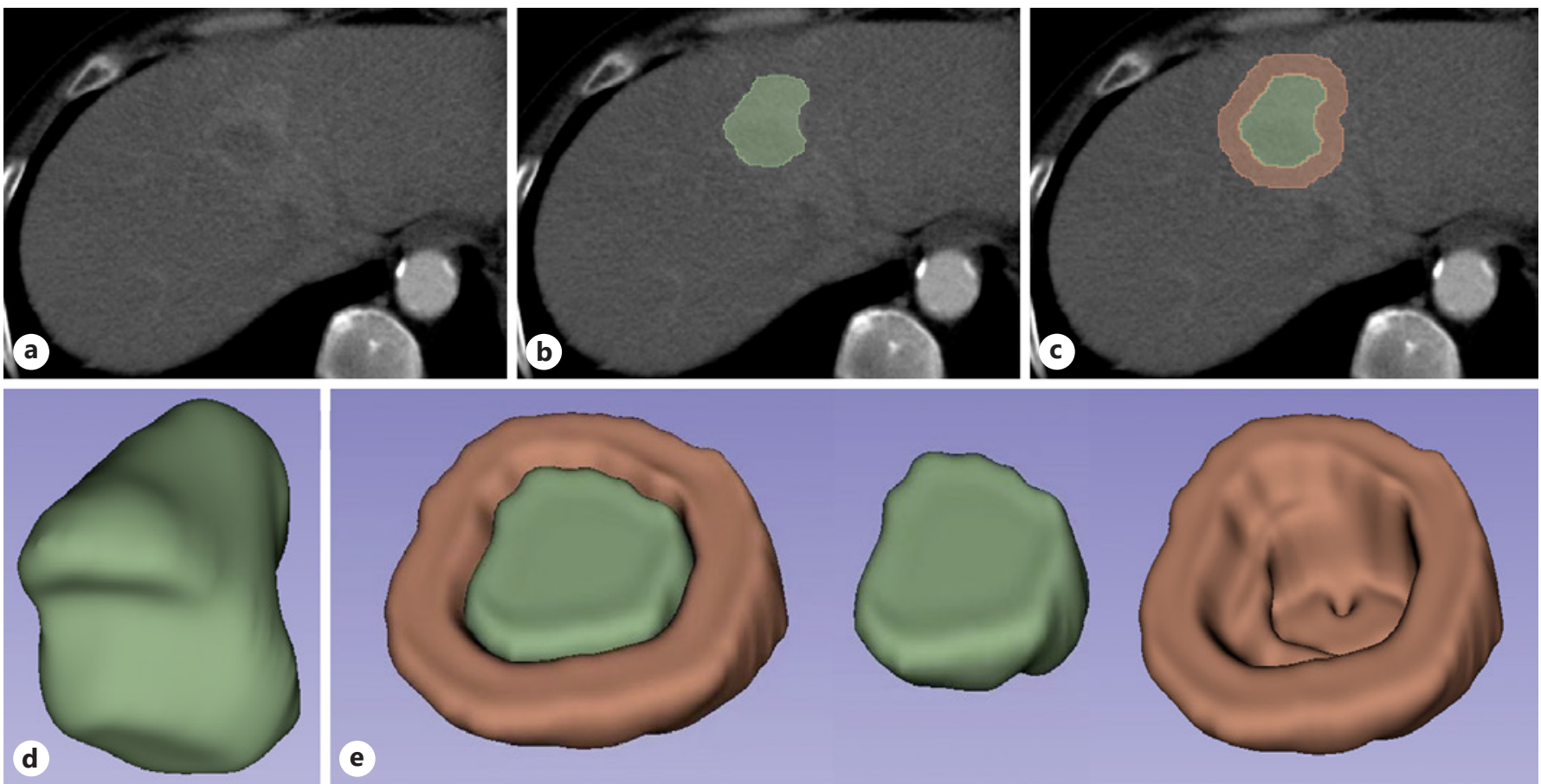

Fig. 2. ROI segmentation for radiomic analysis. First, radiologists checked the lesion on CECT (a). Then, 2 regions that enclosed the contour of the tumor (b) and peritumoral (c) tissues were drawn, respectively. Finally, the computer automatically segmented the 2 regions, which resulted in the identification of the volumetric fea-

and noncontrast phases, respectively (Fig. 2). Finally, there were 4 ROIs identified from those 2 phases after ROI segmentation for each patient. The radiomic features were then extracted from each ROI using the 3D slicer software with an extended plug-in called "PyRadiomics package" plug-in (https://www.radiomics.io/ pyradiomics.html). This plug-in automatically extracted 851 radiomic features from each ROI. The radiomic data from each ROI (main part or expanded rim part at hepatic arterial or noncontrast phase) and image/radiological data from nontargeted lesions (HCC outside the analyzed part) are reported in detail in online suppl. Text 2. Furthermore, to facilitate distinguishing between the 4 radiomic features (i.e., "tumoral ROI at arterial phase," "tumoral ROI at noncontrast phase," "peritumoral ROI at arterial phase," and "peritumoral ROI at noncontrast phase"), these features were, respectively, labeled as "THA," "TNC," "PHA," and "PNC" according to the corresponding tissue and CT phase.

\section{Tumoral and Peritumoral Radiomic Signature}

A total of 355 and 118 targeted lesions were included in the training and internal validation sets, respectively. Significant radiomic features associated with treatment response were identified and selected using the widely used least absolute shrinkage and selection operator (LASSO) method with 10 -fold cross-validation. This method could remove the insignificant features that least affected the objective function and leave those features that significantly associated with treatment response. The tumoral and peritumoral radiomic signature (TPR-signature) was built based on tures of the tumor (ROI tumor, green) and peritumor (ROI peritumor, red). The entire ROI tumor (d); half of the 2 ROIs (e), half of the ROI tumor (green) and ROI peritumor (red), respectively. ROI, region of interest; CECT, contrast-enhanced computed tomography.

significant features from the tumoral and/or peritumoral ROI. The cutoff value was then identified using Youden's index to divide patients into low- and high-risk subgroups (above and below this cutoff).

\section{Construction of the Clinical-Radiomic Nomogram}

Laboratory variables were categorized based on normal reference ranges, including those for ALT ( $\leq 50$ or $>50 \mathrm{U} / \mathrm{mL}$ ), AST $(\leq 40$ or $>40 \mathrm{U} / \mathrm{mL})$, ALB $(\leq 36$ or $>36 \mathrm{~g} / \mathrm{L})$, bilirubin $(\leq 17.1$ or $>17.1 \mathrm{U} / \mathrm{mL})$, and PT $(\leq 13$ or $>13 \mathrm{~s})$. Due to there being no obvious cutoff value, age was evenly separated into 2 categories ( $\leq 60$ or $>60$ years $)$. AFP $(\leq 200$ or $>200 \mathrm{ng} / \mathrm{mL})$ and tumor size $(\leq 5$ or $>5$ $\mathrm{cm}$ ) were categorized based on clinically relevant cutoff values. These clinical variables were subsequently compared by univariate comparison. Statistically significant variables were included along with the TPR-signature in the multivariable logistic regression models. A nomogram including statistically significant factors from the multivariate analysis was developed, and a final model was selected using a backward step-down process, with the Akaike information criterion as a stopping rule.

To use this nomogram, a vertical line was drawn over the graph from the top point row to the bottom of the scores row to obtain the points of each variable. The total points from each intersecting point of the vertical line were then added up to identify the predicted probability. Moreover, an easy-to-use web tool of this nomogram was later designed for public use (see the Results section). 
Table 1. Demographic and clinical characteristics of patients in each cohort

\begin{tabular}{|c|c|c|c|}
\hline Characteristics & $\begin{array}{l}\text { Training cohort } \\
(N=355)\end{array}$ & $\begin{array}{l}\text { Internal validation } \\
\text { cohort } \\
(N=118)\end{array}$ & $\begin{array}{l}\text { External validation } \\
\text { cohort } \\
(N=122)\end{array}$ \\
\hline Age, years & $57[49-65]$ & $56[49-64]$ & $56[47-65]$ \\
\hline Sex (male) & $318(89.6)$ & $103(87.3)$ & $144(93.4)$ \\
\hline Hypertension & $133(37.5)$ & $41(34.7)$ & $49(40.2)$ \\
\hline Diabetes & $126(35.5)$ & $41(34.7)$ & $41(33.6)$ \\
\hline Cirrhosis & $166(46.8)$ & $56(47.5)$ & $55(45.1)$ \\
\hline Hepatitis & $293(82.5)$ & $97(82.2)$ & $100(82.0)$ \\
\hline $\mathrm{HBV}$ & $290(81.7)$ & $96(81.4)$ & $99(81.1)$ \\
\hline $\mathrm{HCV}$ & $3(0.8)$ & $1(0.8)$ & $1(0.8)$ \\
\hline Antiviral treatment & $170(47.9)$ & $52(44.1)$ & $59(48.4)$ \\
\hline $\mathrm{AFP}, \mathrm{ng} / \mathrm{mL}$ & $13.2[4.0-233.9]$ & $18.2[3.9-258.6]$ & $12.7[4.2-285.4]$ \\
\hline$\leq 200$ & $263(74.1)$ & $84(71.2)$ & $84(68.9)$ \\
\hline$>200$ & $92(25.9)$ & $34(28.8)$ & $38(31.1)$ \\
\hline $\mathrm{ALT}, \mathrm{U} / \mathrm{mL}$ & $34.0[23.0-50.1]$ & $33.0[24.0-47.25]$ & $32.0[23.0-51.0]$ \\
\hline$\leq 50$ & $268(75.5)$ & $91(77.1)$ & $90(73.8)$ \\
\hline$>50$ & $87(24.5)$ & $27(22.9)$ & $32(26.2)$ \\
\hline AST, U/mL & $29.0[17.0-44.2]$ & $28.5[17.0-42.0]$ & $27.5[17.0-47.0]$ \\
\hline$\leq 40$ & $249(70.1)$ & $88(74.6)$ & $81(66.4)$ \\
\hline$>40$ & $106(29.9)$ & $30(25.4)$ & $41(33.6)$ \\
\hline $\mathrm{ALB}, \mathrm{g} / \mathrm{L}$ & $40.2[37.0-43.5]$ & $40.6[37.8-43.7]$ & $39.3[36.1-43.1]$ \\
\hline$\leq 36$ & $63(17.7)$ & $13(11.0)$ & $30(24.6)$ \\
\hline$>36$ & $292(82.3)$ & $105(89.0)$ & $92(75.4)$ \\
\hline $\mathrm{TB}, \mu \mathrm{mol} / \mathrm{L}$ & $14.7[11.5-20.0]$ & $15.0[12.4-20.7]$ & $14.4[10.4-18.9]$ \\
\hline$\leq 17.1$ & $230(64.8)$ & $74(62.7)$ & $79(64.8)$ \\
\hline$>17.1$ & $125(35.2)$ & $44(37.3)$ & $43(35.2)$ \\
\hline PT, seconds & $13.5[12.8-14.1]$ & $13.4[12.9-14.0]$ & $13.6[12.9-14.3]$ \\
\hline$\leq 13$ & $123(34.6)$ & $39(33.1)$ & $40(32.8)$ \\
\hline$>13$ & $232(65.4)$ & $79(66.9)$ & $82(67.2)$ \\
\hline ALBI grade $(\mathrm{I}, \mathrm{II})$ & $202 / 153(56.9 / 43.1)$ & $71 / 47(60.2 / 39.8)$ & $63 / 59(51.6 / 48.4)$ \\
\hline Child-Pugh (A, B) & $343 / 12(96.6 / 3.4)$ & $118 / 0(100 / 0)$ & $115 / 7(94.3 / 5.7)$ \\
\hline \multicolumn{4}{|l|}{ BCLC B subclassification } \\
\hline $\mathrm{B} 1$ & $254(71.5)$ & $85(72.0)$ & $82(67.2)$ \\
\hline $\mathrm{B} 2$ & $90(25.3)$ & $33(28.0)$ & $33(27.0)$ \\
\hline B3 & $8(2.3)$ & NA & $6(4.9)$ \\
\hline B4 & $3(0.9)$ & NA & $1(0.8)$ \\
\hline Tumor, $n(\leq 3,>3)$ & $202 / 153(56.9 / 43.1)$ & $70 / 48(59.3 / 40.7)$ & $73 / 49(59.8 / 40.2)$ \\
\hline$\leq 3$ & $202(56.9)$ & $70(59.3)$ & $73(59.8)$ \\
\hline 4 & $81(22.8)$ & $28(23.7)$ & $24(19.7)$ \\
\hline 5 & $70(19.7)$ & $19(16.1)$ & $24(19.7)$ \\
\hline$\geq 6$ & $2(0.6)$ & $1(0.8)$ & $1(0.8)$ \\
\hline Tumor size, $\mathrm{cm}$ & $4.5[3.0-6.5]$ & $5.0[3.0-7.0]$ & $5.1[3.2-7.5]$ \\
\hline \multicolumn{4}{|l|}{ Tumor location } \\
\hline Right lobe & $255(71.8)$ & $83(70.3)$ & $89(73.0)$ \\
\hline Left lobe & $47(13.2)$ & $15(12.7)$ & $21(17.2)$ \\
\hline Segment I & $10(2.8)$ & $5(4.3)$ & $1(0.8)$ \\
\hline Segment IV & $43(12.2)$ & $15(12.7)$ & $11(9.0)$ \\
\hline \multicolumn{4}{|l|}{ Arterial hyperenhancement } \\
\hline Homogeneous & $48(13.5)$ & $16(13.6)$ & $15(12.3)$ \\
\hline Heterogeneous & $262(73.8)$ & $86(72.8)$ & $93(76.2)$ \\
\hline None (hypovascular) & $45(12.7)$ & $16(13.6)$ & $14(11.5)$ \\
\hline
\end{tabular}


Table 1 (continued)

\begin{tabular}{|c|c|c|c|}
\hline Characteristics & $\begin{array}{l}\text { Training cohort } \\
(N=355)\end{array}$ & $\begin{array}{l}\text { Internal validation } \\
\text { cohort } \\
(N=118)\end{array}$ & $\begin{array}{l}\text { External validation } \\
\text { cohort } \\
(N=122)\end{array}$ \\
\hline \multicolumn{4}{|l|}{ Treatment and follow-up } \\
\hline Response to TACE (OR/NR) & $184 / 171(51.8 / 48.2)$ & $61 / 57(51.7 / 48.3)$ & $61 / 61(50.0 / 50.0)$ \\
\hline $\mathrm{CR}$ & $26(7.3)$ & $11(9.3)$ & $8(6.6)$ \\
\hline $\mathrm{PR}$ & $158(44.5)$ & $50(42.4)$ & $53(43.4)$ \\
\hline SD & $137(38.6)$ & $45(38.1)$ & $52(42.6)$ \\
\hline $\mathrm{PD}$ & $34(9.6)$ & $12(10.2)$ & $9(7.4)$ \\
\hline \multicolumn{4}{|l|}{ Rounds of TACE } \\
\hline 1 & $169(47.6)$ & $52(44.1)$ & $66(54.1)$ \\
\hline 2 & $139(39.1)$ & $47(39.8)$ & $41(33.6)$ \\
\hline 3 & $37(10.4)$ & $15(12.7)$ & $12(9.8)$ \\
\hline 4 & $7(2.0)$ & $2(1.7)$ & $2(1.6)$ \\
\hline$\geq 5$ & $3(0.9)$ & $2(1.7)$ & $1(0.8)$ \\
\hline \multicolumn{4}{|l|}{ Embolic materials } \\
\hline Lipiodol & $238(67.04)$ & $86(72.88)$ & $91(74.59)$ \\
\hline Gelatin sponge microparticles & $86(24.23)$ & $20(16.95)$ & $16(13.11)$ \\
\hline Polyvinyl alcohol particles & $20(5.63)$ & $8(6.78)$ & $11(9.02)$ \\
\hline Others & $11(3.10)$ & $4(3.39)$ & $4(3.28)$ \\
\hline \multicolumn{4}{|l|}{ Combined with chemotherapeutic agent } \\
\hline L-OHP & $176(49.58)$ & $52(44.07)$ & $65(53.28)$ \\
\hline $5-\mathrm{FU}$ & $68(19.15)$ & $27(22.88)$ & $20(16.39)$ \\
\hline THP & $46(12.96)$ & $13(11.02)$ & $15(12.30)$ \\
\hline EPI & $33(9.30)$ & $14(11.86)$ & $12(9.84)$ \\
\hline MMC & $14(3.94)$ & $7(5.93)$ & $4(3.28)$ \\
\hline Others & $5(1.41)$ & $2(1.69)$ & $1(0.82)$ \\
\hline None & $13(3.66)$ & $3(2.54)$ & $4(3.28)$ \\
\hline Combined with other treatment (no, yes) & $307 / 48(86.5 / 13.5)$ & $100 / 18(84.7 / 15.2)$ & $102 / 20(83.6 / 16.4)$ \\
\hline Supportive care & $307(86.5)$ & $100(84.7)$ & $102(83.6)$ \\
\hline Sorafenib or PD1 & $42(11.8)$ & $14(11.9)$ & $19(15.6)$ \\
\hline Ablation & $6(1.7)$ & $4(3.4)$ & $1(0.8)$ \\
\hline Date to follow-up CT, days & $47[40-53]$ & $48[40-54]$ & $47[41-55]$ \\
\hline Median follow-up, months & $24.0[17.0-41.0]$ & $23.0[14.0-36.3]$ & $25.0[13.0-40.2]$ \\
\hline
\end{tabular}

Data are presented as median [interquartile range] or number (percent). HBV/HCV, hepatitis B/C virus; AFP, alpha-fetoprotein; ALT, alanine aminotransferase; AST, aspartate aminotransferase; ALB, albumin; TB, total bilirubin; PT, prothrombin time; ALBI, albumin-bilirubin; OR, objective response; NR, nonresponsive; TACE, transarterial chemoembolization; $\mathrm{CR}$, complete response; $\mathrm{PR}$, partial response; $\mathrm{SD}$, stable disease; $\mathrm{PD}$, progressive disease; BCLC, Barcelona Clinic Liver Cancer System; L-OHP, oxaliplatin; 5-FU, fluorouracil; THP, pirarubicin; EPI, epirubicin; MMC, mitomycin.

Statistical Analysis

The primary endpoint of interest was the actual treatment response status to initial TACE, and the second endpoint of interest was the OS. The former was used to establish a clinical-radiomic nomogram for predicting treatment response. The performance of this nomogram was evaluated using a calibration curve and the area under the receiver operating characteristic curve (AUC). The AUCs were designed to assess the nomogram's performance with survival outcomes. To assess OS across different predicted treatment response statuses, survival curves were constructed using the Kaplan-Meier method and compared with the log-rank test. Univariate and multivariate Cox proportional hazards regression model analyses were performed to identify potential prognostic factors for OS. Statistical analyses were carried out using R software with the "survival" and "rms" packages (http://www.r-project. org). A $p$ value $<0.05$ was considered statistically significant.

\section{Results}

\section{Demographic and Clinical Characteristics of Patients}

The demographic and clinical characteristics of patients with intermediate-stage HCC in the training $(N=$ $355)$, internal validation $(N=118)$, and external valida- 
Table 2. The comparison of 7 different radiomic signatures based on tumoral (main part) and/or peritumoral (expanded rim part) radiomic features

\begin{tabular}{|c|c|c|c|c|c|c|c|c|c|}
\hline $\begin{array}{l}\text { Radiomic models based on } \\
\text { different parts }\end{array}$ & $\begin{array}{l}\text { ROIs, } \\
n\end{array}$ & \multicolumn{4}{|c|}{ Training set $(N=355)$} & \multicolumn{4}{|c|}{ Testing set $(N=118)$} \\
\hline Peritumor (5 mm) & 2 & 56.5 & 65.5 & 60.8 & $0.59[0.45-0.71]$ & 54.1 & 54.4 & 54.2 & $0.55[0.46-0.68]$ \\
\hline Peritumor $(10 \mathrm{~mm})$ & 2 & 59.8 & 61.4 & 60.6 & $0.59[0.47-0.65]$ & 50.8 & 52.6 & 51.7 & $0.53[0.45-0.63]$ \\
\hline Peritumor (20 mm) & 2 & 59.2 & 56.1 & 57.7 & $0.56[0.49-0.64]$ & 49.2 & 52.6 & 50.8 & $0.52[0.42-0.64]$ \\
\hline Tumor + peritumor $(20 \mathrm{~mm})$ & 4 & 81.2 & 77.7 & 79.4 & $0.79[0.76-0.84]$ & 82.4 & 68.9 & 75.4 & $0.75[0.65-0.83]$ \\
\hline
\end{tabular}

ROIs, regions of interest; AUC, area under the receiver operating characteristic curve.

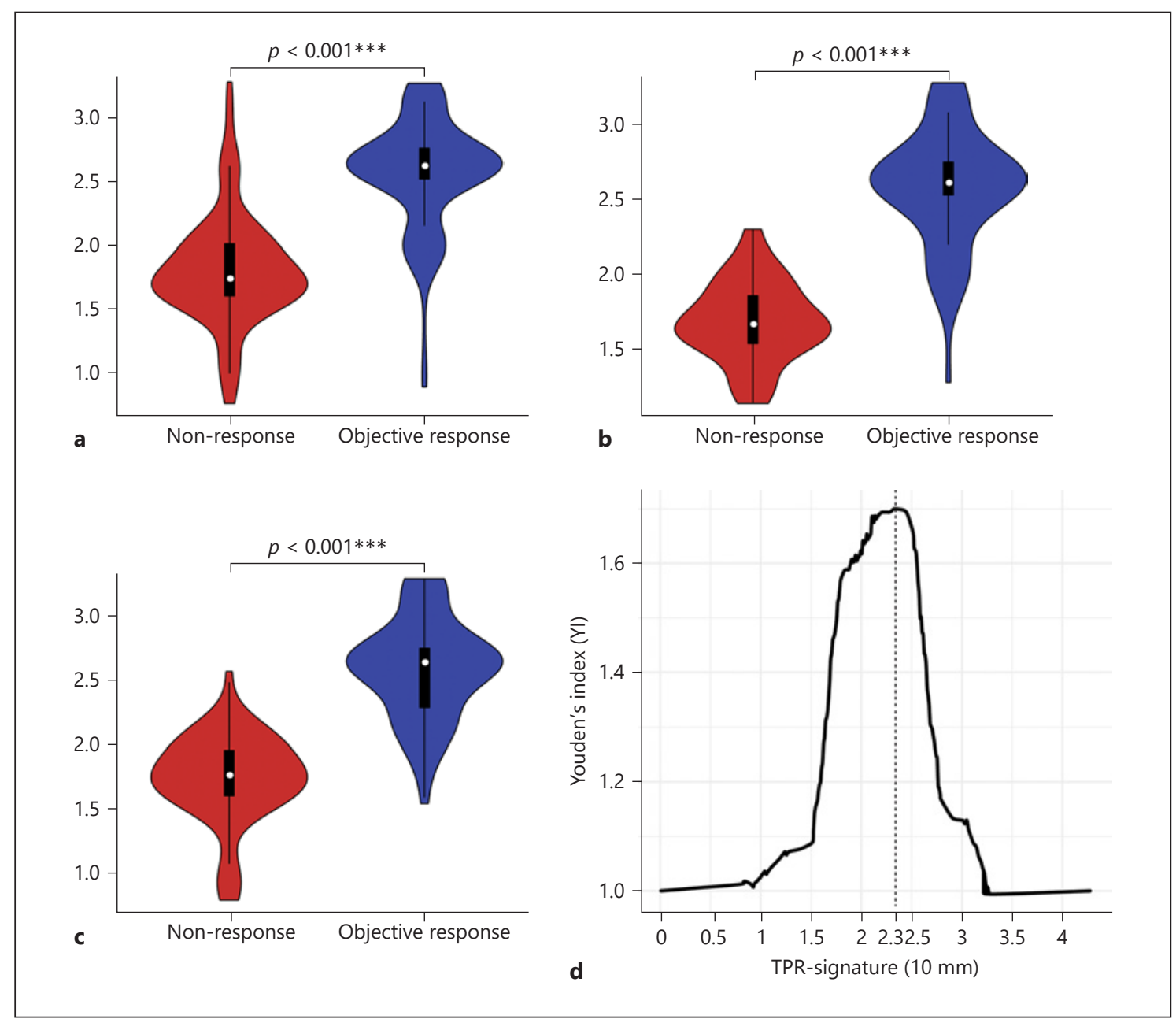

Fig. 3. The distribution of the TPR-signature between the objective response and nonresponse patients. Training cohort (a); internal validation cohort (b); external validation cohort (c); and cutoff point identified with Youden's index (d). ${ }^{*} p<0.05$; ${ }^{* *} p<0.01$; and ${ }^{* * *} p<0.001$. TPR, tumoral and peritumoral radiomic. 


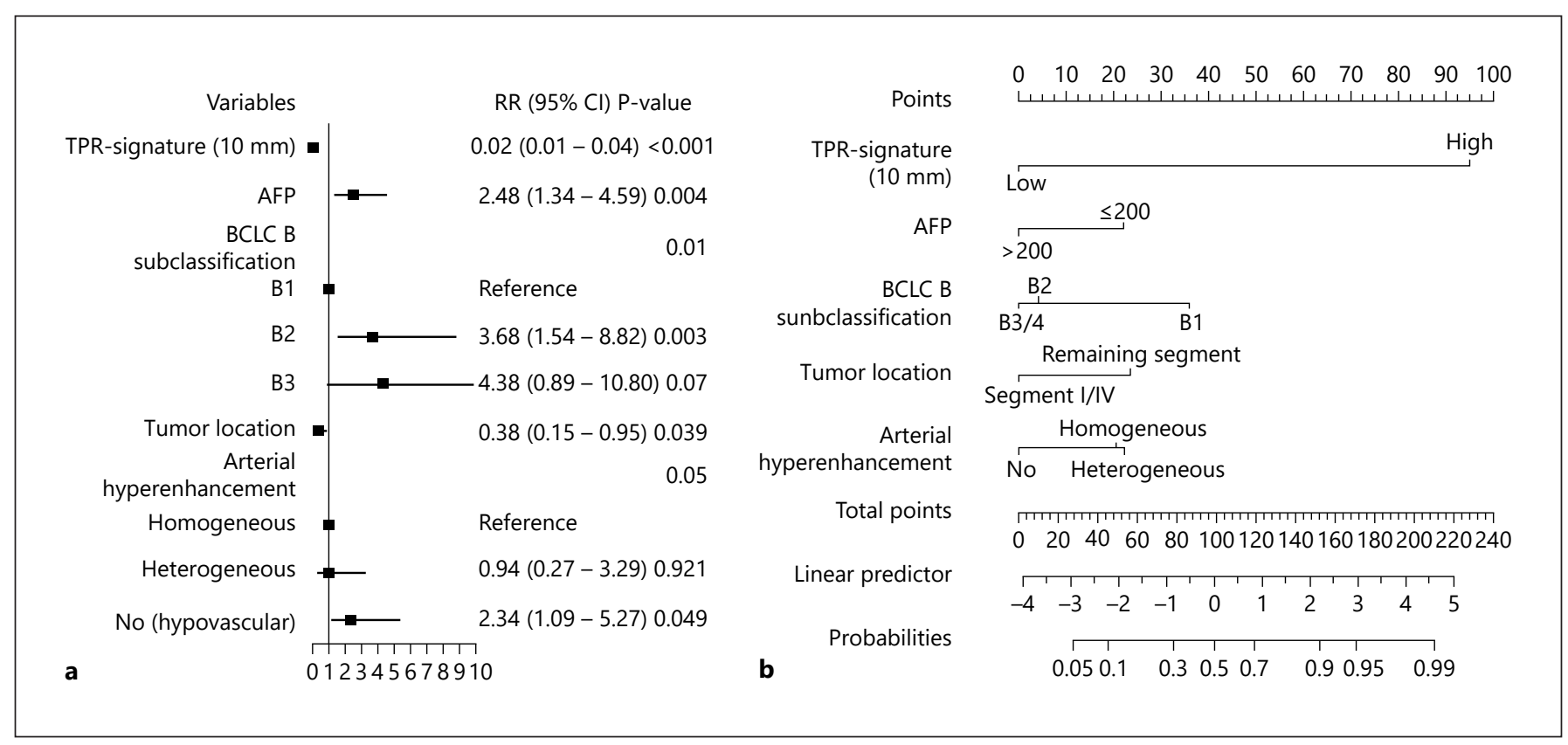

Fig. 4. Forest plot of significant factors and the CR model. Forest plot of significant factors of objective response with a multivariate regression model (a); a nomogram scaled by the coefficient of each factor (b). CR, clinical radiomic; TPR, tumoral and peritumoral radiomic; AFP, alpha-fetoprotein; BCLC B, Barcelona Clinic Liver Cancer staging classification $\mathrm{B}$.

tion $(N=122)$ cohorts are summarized in Table 1 . The median age of the training cohort was 57 years, 318 patients were male, and 166 patients were diagnosed with cirrhosis. Most patients were Child-Pugh A (96.6\%) and BCLC stage B1 (71.5\%). The vast majority $(81.7 \%)$ of the patients were hepatitis B surface antigen positive, and $48 \%$ had received antiretroviral therapy. Similar baseline characteristics of patients with intermediate-stage HCC were observed in both the internal and external validation cohorts. There were no significant differences in terms of patients' characteristics between the training and validation cohorts (online suppl. Table 1).

Over half of the patients in each cohort had an objective response to initial TACE, with a partial response rate of $44.5,42.4$, and $43.4 \%$ in the training, internal, and external validation cohorts, respectively. The median time from initial TACE to first follow-up CECT was approximately 47 days (range: 40-55 days). Median follow-up time ranged between 23 and 26 months in the 3 patient cohorts.

\section{Radiomic Analysis}

A total of 851 radiomic features were extracted from each ROI based on pre-TACE CECT imaging. Then, 7 different models were built based on corresponding features using LASSO and compared using the AUC value (Table 2). The best performance of TPR-signature, which was based on 3,404 radiomic features from the tumoral ROI and peritumoral $(10 \mathrm{~mm})$ ROI, was identified and selected for further analysis. The TPR-signature included 18 radiomic features, 17 of which were selected from tumoral ROI at the hepatic arterial and noncontrast phases (14 vs. 3 , respectively), with the remaining 1 feature coming from the peritumoral ROI at the hepatic arterial phase (online suppl. Table 2 and online suppl. Fig. 1). The TPRsignature all showed a significant difference between the objective response and nonresponsive groups $(p<0.001)$ (Fig. $3 a-c)$. According to the cutoff value of 2.3, the patients could be divided into 2 subgroups (high and low likelihood of objective response) (Fig. 3d).

\section{Clinical-Radiomic Nomogram}

In the training cohort, 8 significant predictors, including the TPR-signature and 7 clinical variables (antiviral treatment, AFP, BCLC B subclassification, tumor number, tumor size, tumor location, and arterial hyperenhancement), proved to be significant during univariate comparison (online suppl. Table 3). Based on the results 

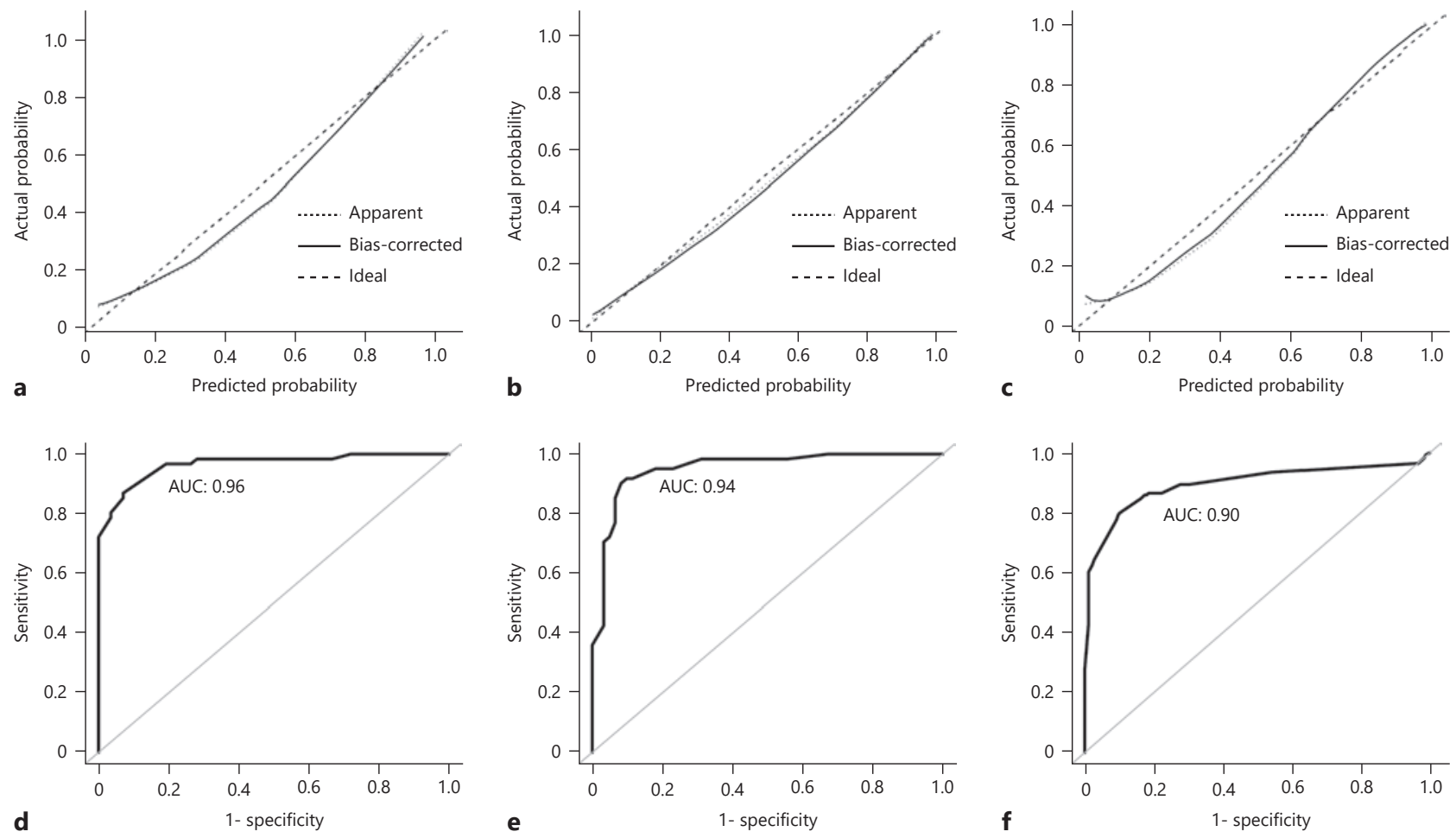

Fig. 5. The calibration curve demonstrating predictions from the model to the actual observed probability. Training cohort (a); internal validation cohort (b); and external validation cohort (c). The receiver operation characteristic curve for predicting objective response to initial TACE in the training (d), internal validation (e), and external validation cohorts (f). TACE, transarterial chemoembolization; AUC, area under the receiver operating characteristic curve.

of multivariate analyses with regression models, 5 independent predictors were selected and taken into the final model (Fig. 4a and online suppl. Table 3). Finally, a clinical-radiomic nomogram was developed to predict the treatment response to initial TACE (Fig. 4b). This nomogram is available as a web tool for public use (online suppl. Fig. 2, https://rc9u60.axshare.com/nomogram.html).

\section{Nomogram Performance}

The performance of this clinical-radiomic nomogram was tested using a calibration curve and AUCs. The calibration plot for the probability of objective response demonstrated optimal agreement between the predicted probabilities computed by the nomogram and actual observations among the 3 patient cohorts (Fig. $5 \mathrm{a}-\mathrm{c}$ ). The discrimination ability of the nomogram was measured by bootstrap-corrected AUCs. The AUCs of the training, internal validation, and external validation cohorts were $0.96,0.94$, and 0.90 , respectively (Fig. $5 d-f$ ).

\section{Evaluation of OS}

The median OS for all patients in the external validation cohort was 27.0 months (range: $23.3-30.7$ months, 95\% confidence interval $[\mathrm{CI}]$ ). Significant differences $(p<0.001)$ in OS were observed between the objective TACE response and nonresponsive groups during both treatment and with the predicted models $(p<0.001)$ (Fig. 6a, b). Univariate and multivariate analyses were performed to identify potential prognostic factors for OS using the Cox proportional hazards regression model. The treatment response status predicted by the clinicalradiomic nomogram (hazard ratio [HR]: $2.43 ; 95 \% \mathrm{CI}$ : $1.60-3.69 ; p<0.001$ ) was an independent prognostic factor for OS. Other prognostic factors included AFP ( $p=$ $0.014)$, BCLC B subclassification $(p<0.001)$, arterial hyperenhancement $(p=0.029)$, and salvage treatments after failure of TACE $(p=0.047)$ (online suppl. Table 4). In addition, predicted treatment response status demonstrated a statistically significant ability to discriminate be- 


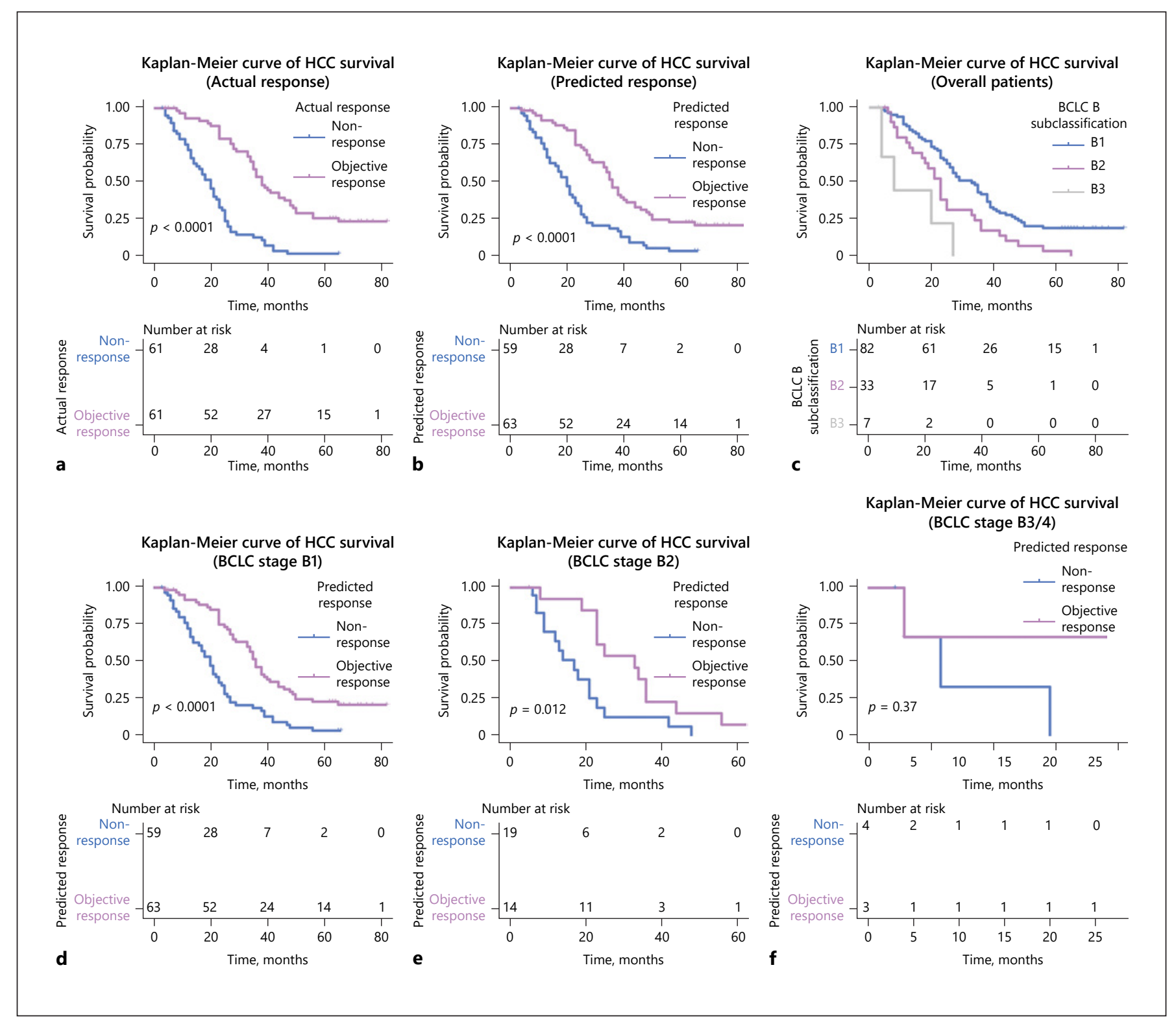

Fig. 6. The Kaplan-Meier curve for intermediate-stage HCC survival. Actual response status (a) and predicted response status by the CR model (b); the Kaplan-Meier curves for OS for each BCLC $\mathrm{B}$ subclassification in the external validation cohort. Overall pa- tients (c), BCLC stage B1 (d), BCLC stage B2 (e), and BCLC stage $\mathrm{B} 3 / 4$ (f). HCC, hepatocellular carcinoma; $\mathrm{CR}$, clinical radiomic; OS, overall survival; BCLC B, Barcelona Clinic Liver Cancer staging classification $\mathrm{B}$. tween Kaplan-Meier curves for survival outcome within each BCLC B subclassification in the external validation and primary cohorts (Fig. $6 \mathrm{c}-\mathrm{f}$ and online suppl. Fig. 3, respectively).

\section{Discussion}

This study developed and validated a CR model for predicting the treatment response of initial TACE in patients with intermediate-stage HCC, based on retrospective data from 595 patients at multiple centers. This CR model integrated a radiomic signature with 4 clinical fac- 
tors: AFP, BCLC B subclassification, tumor location, and arterial hyperenhancement. In addition, the treatment response predicted by our CR model was an independent prognostic factor for OS. The CR model could accurately stratify patients with intermediate-stage (BCLC B and its substages) HCC into 2 prognostic subgroups with significantly different OS. The patients for whom an objective response was predicted were advised to repeat TACE. Conversely, for predicted TACE nonresponders, OS could be significantly improved if consideration was given to other therapies such as sorafenib [35], lenvatinib alone [36], or lenvatinib followed by selective TACE [37].

The TPR-signature which integrated various radiomic features was one of the most important components in predicting treatment response using our CR model. Notably, most of the significant radiomic features were derived from ROIs at the hepatic arterial phase. This corresponded with previous studies that showed that a difference in extracellular volume and blood flow between HCC tissue and nonneoplastic liver tissue during the arterial phase could give rise to unique radiological features [38-40]. A recent meta-analysis also demonstrated similar results with the utilization of arterial phase imaging being related to a higher degree of sensitivity [41].

Previous studies have focused on the primary tumor without taking peritumoral tissue into account for the analysis [42]. In our study, we focused on the potential characteristics of both tumor tissue and peritumoral tissue and explored the influence of the different size of the peritumoral tissue (expanded rim part) on the radiomic calculation. Previous studies on glioma [43], breast cancer [44], and lung cancer $[45,46]$ revealed that the peritumoral tissue 5-20 $\mathrm{mm}$ away from tumor has a close relationship to tumor prognosis. In our study, we found that the TPR-signature $(10 \mathrm{~mm})$ based on tumoral and peritumoral $(10 \mathrm{~mm})$ ROIs had the best performance in the training set (AUC: 0.85 ; 95\% CI: 0.82-0.89) and the testing set (AUC: $0.81 ; 95 \%$ CI: 0.75-0.84). A quite similar AUC value of TPR-signature (5 mm) (AUC: 0.84 ) was observed in the training set, but its range of $95 \% \mathrm{CI}$ was larger and the AUC value (AUC: 0.79) was lower in the testing set. Therefore, the TPR-signature $(10 \mathrm{~mm})$ was identified and selected for further analysis in this study. Although there was only one peritumoral radiomic feature in the TPR-signature, the peritumoral stroma, which was enriched in inflammatory cells, especially monocytes or/and macrophages [47], was considered to be part of the HCC tissue. Peritumoral monocytes might promote the upregulation of autophagy in tumor cells by secreting tumor necrosis factors and interleukin $1 \beta$ at the invading edge region of the HCC, leading to more aggressive cancer progression [48]. Peritumoral hepatic macrophages have been shown to increase with a decrease in nitric oxide synthase 2 expression and an increase in human leukocyte antigen-DR isotype-positive cells, which upregulates $\mathrm{T}$ lymphocytes by producing a variety of cytokines and chemokines [49]. The significant humoral cytokine response observed in peritumoral regions indicates that shifts to immune-suppressive responses might promote HCC venous metastases [50]. However, the radiomic signatures of peritumoral tissue are of less importance than those of the cancer nest $[51,52]$ due to their heterogeneity and the lack of algorithmic standardization. In our study, some tumors were located deep inside the liver, while others were close to Glisson's capsule, leading to a primary difference in the peritumoral ROIs. Based on biological and clinical factors, the size of the peritumoral region varied, and it would be inappropriate to obtain the peritumoral region by setting a consistent distance from the tumor. Thus, further evaluation of the radiomic signatures of peritumoral tissues and the establishment of an individualized size of the peritumoral region can help to describe differences between superficial and deep HCC.

The data in this study confirmed that previously reported clinical predictors, such as tumor location and AFP, could affect treatment response to TACE, and they were incorporated into the CR model. Tumor location (segments I and IV) was related to poor treatment response to TACE. TACE can inhibit HCC progression by cutting off the tumor blood supply. However, segments I and IV have multiple arterial branches originating from the left and right hepatic arteries, with frequent intraparenchymal anastomoses $[53,54]$, which can provide a partial or total blood supply to the tumor, leading to nonresponse to TACE and tumor recurrence [55]. Moreover, AFP level has been identified as one of the most significant prognostic factors [56], and it has been used as an independent OS predictor for patients who undergo TACE [57]. The AFP level can be a moderate predictor for evaluating the tumor response before TACE because patients with a high AFP ( $>200 \mathrm{ng} / \mathrm{mL})$ have a lower TACE response than their counterparts [58].

Several factors (e.g., tumor size) were not incorporated into our CR model; however, these factors could indirectly contribute to predicting treatment response and should not be ignored. Previous studies have observed that patients with tumors $>5 \mathrm{~cm}$ generally have a worse treatment response rate [19]. Our study also revealed that tumor size was significantly associated with the TACE response $(p=0.003)$ during univariate analysis, but not 
in multivariate analysis, suggesting that tumor size was not an independent factor useful for building the $\mathrm{CR}$ model. In this respect, it can be said that BCLC B subclassification had a similar function as tumor size $(\leq 5$ and $>5$ $\mathrm{cm}$ ) classification in the CR model because the distinction between BCLC stage B1 and B2 mainly depends on the Milan criteria, which takes the tumor size into account. Therefore, tumor size was a component, albeit indirectly, of our CR model.

In addition to treatment response, the performance of our model was evaluated by OS. We found that treatment response to initial TACE predicted by our CR model was an important prognostic factor of OS. In fact, significant difference in OS occurred between patients with objective response and nonresponse, either when the response was predicted by the model or observed by a physician. The median OS for all patients was 27.0 months, which exceeded the median OS of 19.4 months reported by Lencioni et al. [59]. This expected difference could be attributed to the improved process for selecting ideal TACE candidates over time [60]. The utility of the CR model was convincingly supported by the significant difference in OS observed within the BCLC substages B1 $(p<0.001)$ and B2 $(p=$ 0.012 ) of the external validation cohort. Even for BCLC stage $\mathrm{B} 3 / 4$, patients with predicted nonresponse had a shorter OS than their counterparts although the small sample size might have contributed to an underpowered analysis of this patient group.

Despite the importance of the developed model, this study has several limitations. Firstly, this was a retrospective study, and several clinical variables could not be measured. For example, injected drugs and their dosages during TACE were not homogenous across patients in the training, internal validation, and external validation cohorts. Secondly, selecting the radiomic data from the largest parts of individual HCC tumor and the radiological features from other nontargeted lesions before the initial TACE might have affected the predictive outcomes of models. Therefore, it is feasible for clinicians to weigh the pros and cons in order to increase convenience and decrease workloads. Thirdly, while the number of TACE treatments can influence OS, patients who had an objective response to initial TACE had a better OS, regardless of the number of rounds of TACE, which is very useful information for clinicians in selecting patients. As predictive models based on radiomics of multiple rounds of TACE are created with greater sophistication, further studies exploring the relationship of the number of TACE rounds and survival can be designed. At present, our model can help clinicians select targeting agents in advance so that the appropriate treatment for HCC in patients can be chosen before initial TACE or immediately after failure. Fourthly, our CR model was developed and validated in a setting where most patients had HBV-related HCC. Therefore, with regard to the possible etiologybased differences in cancer biology, this model should be validated in a Western context, where hepatitis $\mathrm{C}$ and alcohol abuse are more common [61]. Because of the limited number of patients with BCLC stage B3/4, the corresponding results need to be tested by a larger patient cohort with BCLC B3/4. Finally, prospective data including patients with various etiologies for chronic liver disease and HCC would help evaluate and validate this CR model.

\section{Conclusion}

We have developed and validated a CR model to predict preoperative treatment response in patients with intermediate-stage HCC treated with initial TACE. The model had an excellent predictive performance and could be a powerful tool to assist clinicians in selecting patients who would optimally benefit from first TACE.

\section{Acknowledgements}

We would like to thank the Hangzhou Xixuan Health technology Co., Ltd. for their assistance in developing and testing different models. Thanks are due to Yifan Tong, Tunan Yu, and Guangyi Jiang for their efforts spent in the data collection and patient follow-up. We are also grateful to our radiological colleagues for their assistance in checking the radiological information.

\section{Statement of Ethics}

This study was conducted at 3 medical institutions in accordance with the Declaration of Helsinki. The study protocol/ethics was approved by each Institutional Review Board (KY20190520-1, 2019HCC024, and 20190527). Patients' informed consent was waived or not required, as this is a retrospective study, and the analysis used anonymous clinical data that were obtained after the patient had agreed to treatment with written consent.

\section{Conflict of Interest Statement}

The authors have no conflicts of interest to declare. 


\section{Funding Sources}

This work was supported by the National Natural Science Foundation of China (Nos. 81800540 and 81827804), the Scientific Research Projects of Zhejiang Education Department (No. Y201941406), the Opening Fund of Engineering Research Center of Cognitive Healthcare of Zhejiang Province (No. 2018KFJJ09), and the Zhejiang Clinical Research Center of Minimally Invasive Diagnosisand Treatment of AbdominalDiseases(No.2018E50003).

\section{Author Contributions}

M.Y.C., J.S.C., and J.H.H. performed the described studies, analyzed the data, and prepared the manuscript. W.T., S.J.L., S.J., J.L., C.H.T., J.L.S., and B.Z. collected and checked data. M.Y.C., J.S.C., and X.J.C. developed and validated the methodology. J.W., C.P., M.K., A.A., F.T., P.S.S., V.M.Z., and T.K. advised on the study design and prepared the manuscript. All authors read and approved the final manuscript.

\section{References}

1 Bray F, Ferlay J, Soerjomataram I, Siegel RL, Torre LA, Jemal A. Global cancer statistics 2018: GLOBOCAN estimates of incidence and mortality worldwide for 36 cancers in 185 countries. CA Cancer J Clin. 2018;68(6):394424.

2 Miller KD, Goding Sauer A, Ortiz AP, Fedewa SA, Pinheiro PS, Tortolero-Luna G, et al. Cancer statistics for Hispanics/Latinos, 2018. CA Cancer J Clin. 2018;68(6):425-45.

3 Siegel RL, Miller KD, Jemal A. Cancer statistics. CA Cancer J Clin. 2019;2019(69):7-34.

4 Omata M, Cheng AL, Kokudo N, Kudo M, Lee JM, Jia J, et al. Asia-Pacific clinical practice guidelines on the management of hepatocellular carcinoma: a 2017 update. Hepatol Int. 2017;11(4):317-70.

5 Kanwal F, Befeler A, Chari RS, Marrero J, Kahn J, Afdhal N, et al. Potentially curative treatment in patients with hepatocellular cancer: results from the liver cancer research network. Aliment Pharmacol Ther. 2012;36(3): 257-65.

6 Cai X. Laparoscopic liver resection: the current status and the future. Hepatobiliary Surg Nutr. 2018;7(2):98-104.

7 Kudo M, Han G, Finn RS, Poon RT, Blanc JF, Yan L, et al. Brivanib as adjuvant therapy to transarterial chemoembolization in patients with hepatocellular carcinoma: a randomized phase III trial. Hepatology. 2014;60(5):1697707.

8 European Association for the Study of the Liver. EASL Clinical Practice Guidelines: management of hepatocellular carcinoma. J Hepatol. 2018;69:182-236.

9 Heimbach JK, Kulik LM, Finn RS, Sirlin CB, Abecassis MM, Roberts LR, et al. AASLD guidelines for the treatment of hepatocellular carcinoma. Hepatology. 2018;67(1):358-80.

10 Marrero JA, Kulik LM, Sirlin CB, Zhu AX, Finn RS, Abecassis MM, et al. Diagnosis, staging, and management of hepatocellular carcinoma: 2018 practice guidance by the American Association for the Study of Liver Diseases. Hepatology. 2018;68(2):723-50.

11 Llovet JM, Real MI, Montana X, Planas R, Coll $\mathrm{S}$, Aponte J, et al. Arterial embolisation or chemoembolisation versus symptomatic treatment in patients with unresectable hepatocellular carcinoma: a randomised controlled trial. Lancet. 2002;359:1734-9.
12 Hiraoka A, Kumada T, Kudo M, Hirooka M, Koizumi Y, Hiasa Y, et al. Real-life practice experts for HCCSG, group HCC: hepatic function during repeated TACE procedures and prognosis after introducing sorafenib in patients with unresectable hepatocellular carcinoma: multicenter analysis. Dig Dis. 2017; 35:602-10.

13 Arizumi T, Ueshima K, Minami T, Kono M, Chishina $\mathrm{H}$, Takita $\mathrm{M}$, et al. Effectiveness of sorafenib in patients with transcatheter arterial chemoembolization (TACE) refractory and intermediate-stage hepatocellular carcinoma. Liver cancer. 2015;4(4):253-62.

14 Lin H, Lin L, Wang G, Zuo N, Zhan Z, Xie S, et al. Label-free classification of hepatocellular-carcinoma grading using second harmonic generation microscopy. Biomed Opt Express. 2018;9(8):3783-93.

15 Ogasawara S, Chiba T, Ooka Y, Kanogawa N, Motoyama T, Suzuki E, et al. Efficacy of sorafenib in intermediate-stage hepatocellular carcinoma patients refractory to transarterial chemoembolization. Oncology. 2014; 87(6):330-41.

16 Piscaglia F, Ogasawara S. Patient selection for transarterial chemoembolization in hepatocellular carcinoma: importance of benefit/ risk assessment. Liver Cancer. 2018;7(1):10419.

17 Sieghart W, Hucke F, Peck-Radosavljevic M. Transarterial chemoembolization: modalities, indication, and patient selection. J Hepatol. 2015;62(5):1187-95.

18 Vesselle G, Quirier-Leleu C, Velasco S, Charier F, Silvain C, Boucebci S, et al. Predictive factors for complete response of chemoembolization with drug-eluting beads (DEBTACE) for hepatocellular carcinoma. Eur Radiol. 2016;26(6):1640-8.

19 Purcell Y, Sartoris R, Paradis V, Vilgrain V, Ronot M. Influence of pretreatment tumor growth rate on objective response of hepatocellular carcinoma treated with transarterial chemoembolization. J Gastroenterol Hepatol. 2020 Feb;35(2):305-18.

20 Hucke F, Pinter M, Graziadei I, Bota S, Vogel W, Müller C, et al. How to STATE suitability and START transarterial chemoembolization in patients with intermediate stage hepatocellular carcinoma. J Hepatol. 2014;61(6):128796.
21 Hucke F, Sieghart W, Pinter M, Graziadei I, Vogel W, Müller C, et al. The ART-strategy: sequential assessment of the ART score predicts outcome of patients with hepatocellular carcinoma re-treated with TACE. J Hepatol. 2014;60(1):118-26.

22 Sieghart W, Hucke F, Pinter M, Graziadei I, Vogel W, Müller C, et al. The ART of decision making: retreatment with transarterial chemoembolization in patients with hepatocellular carcinoma. Hepatology. 2013;57(6):2261-73.

23 Kadalayil L, Benini R, Pallan L, O’Beirne J, Marelli L, Yu D, et al. A simple prognostic scoring system for patients receiving transarterial embolisation for hepatocellular cancer. Ann Oncol. 2013;24:2565-70.

24 Park Y, Kim SU, Kim BK, Park JY, Kim DY, Ahn SH, et al. Addition of tumor multiplicity improves the prognostic performance of the hepatoma arterial-embolization prognostic score. Liver Int. 2016;36:100-7.

25 Cappelli A, Cucchetti A, Cabibbo G, Mosconi C, Maida M, Attardo S, et al. Refining prognosis after trans-arterial chemo-embolization for hepatocellular carcinoma. Liver Int. 2016; 36:729-36.

26 Han G, Berhane S, Toyoda H, Bettinger D, Elshaarawy O, Chan AWH, et al. Prediction of survival among patients receiving transarterial chemoembolization for hepatocellular carcinoma: a response-based approach. Hepatology. $2020 \mathrm{Jul} ; 72: 198-212$.

27 Liu Z, Wang S, Dong D, Wei J, Fang C, Zhou $\mathrm{X}$, et al. The applications of radiomics in precision diagnosis and treatment of oncology: opportunities and challenges. Theranostics. 2019;9(5):1303-22.

28 Freedman AN, Seminara D, Gail MH, Hartge P, Colditz GA, Ballard-Barbash R, et al. Cancer risk prediction models: a workshop on development, evaluation, and application. J Natl Cancer Inst. 2005;97(10):715-23.

29 Pinato DJ, Sharma R, Allara E, Yen C, Arizumi T, Kubota K, et al. The ALBI grade provides objective hepatic reserve estimation across each BCLC stage of hepatocellular carcinoma. J Hepatol. 2017;66(2):338-46.

30 Johnson PJ, Berhane S, Kagebayashi C, Satomura S, Teng M, Reeves HL, et al. Assessment of liver function in patients with hepatocellular carcinoma: a new evidence-based approachthe ALBI grade. J Clin Oncol. 2015;33:550-8. 
31 Bolondi L, Burroughs A, Dufour JF, Galle PR, Mazzaferro V, Piscaglia F, et al. Heterogeneity of patients with intermediate (BCLC B) hepatocellular carcinoma: proposal for a subclassification to facilitate treatment decisions. Semin Liver Dis. 2012;32(4):348-59.

32 Xu X, Zhang HL, Liu QP, Sun SW, Zhang J, Zhu FP, et al. Radiomic analysis of contrastenhanced CT predicts microvascular invasion and outcome in hepatocellular carcinoma. J Hepatol. 2019;70(6):1133-44.

33 Banerjee S, Wang DS, Kim HJ, Sirlin CB, Chan MG, Korn RL, et al. A computed tomography radiogenomic biomarker predicts microvascular invasion and clinical outcomes in hepatocellular carcinoma. Hepatology. 2015;62(3):792-800.

34 Wang Q, Xia D, Bai W, Wang E, Sun J, Huang $\mathrm{M}$, et al. Development of a prognostic score for recommended TACE candidates with hepatocellular carcinoma: a multicentre observational study. J Hepatol. 2019;70(5):893903.

35 Berger-Richardson D, Chesney TR, Englesakis M, Govindarajan A, Cleary SP, Swallow CJ. Trends in port-site metastasis after laparoscopic resection of incidental gallbladder cancer: a systematic review. Surgery. 2017; 161(3):618-27.

36 Kudo M, Ueshima K, Chan S, Minami T, Chishina $\mathrm{H}$, Aoki $\mathrm{T}$, et al. lenvatinib as an initial treatment in patients with intermediatestage hepatocellular carcinoma beyond upto-seven criteria and Child-Pugh A liver function: a proof-of-concept study. Cancers. 2019 Jul 31:11(8):1084.

37 Kudo M. A new treatment option for intermediate-stage hepatocellular carcinoma with high tumor burden: initial lenvatinib therapy with subsequent selective TACE. Liver Cancer. 2019;8(5):299-311.

38 Chou R, Cuevas C, Fu R, Devine B, Wasson $\mathrm{N}$, Ginsburg A, et al. Imaging techniques for the diagnosis of hepatocellular carcinoma: a systematic review and meta-analysis. Ann Intern Med. 2015;162(10):697-711.

39 Choi JY, Lee JM, Sirlin CB. CT and MR imaging diagnosis and staging of hepatocellular carcinoma: part I. Development, growth, and spread: key pathologic and imaging aspects. Radiology. 2014;272(3):635-54.

40 Stevens WR, Johnson CD, Stephens DH, Batts KP. CT findings in hepatocellular carcinoma: correlation of tumor characteristics with causative factors, tumor size, and histologic tumor grade. Radiology. 1994;191(2):531-7.
41 Yamashita Y, Mitsuzaki K, Yi T, Ogata I, Nishiharu T, Urata J, et al. Small hepatocellular carcinoma in patients with chronic liver damage: prospective comparison of detection with dynamic MR imaging and helical CT of the whole liver. Radiology. 1996;200(1):7984.

42 Lee IC, Hung YW, Liu CA, Lee RC, Su CW, Huo TI, et al. A new ALBI-based model to predict survival after transarterial chemoembolization for BCLC stage B hepatocellular carcinoma. Liver Int. 2019 Sep;39(9):170412.

43 Sun X, Pang P, Lou L, Feng Q, Ding Z, Zhou $J$. Radiomic prediction models for the level of Ki-67 and p53 in glioma. J Int Med Res. 2020; 48(5):300060520914466.

44 Zhou J, Zhang Y, Chang KT, Lee KE, Wang $\mathrm{O}, \mathrm{Li}$ J, et al. Diagnosis of benign and malignant breast lesions on DCE-MRI by using radiomics and deep learning with consideration of peritumor tissue. J Magn Reson Imaging. 2020;51(3):798-809.

45 Dou TH, Coroller TP, van Griethuysen JJM, Mak RH, Aerts HJWL. Peritumoral radiomics features predict distant metastasis in locally advanced NSCLC. PLoS One. 2018;13(11): e0206108.

46 Wang X, Zhao X, Li Q, Xia W, Peng Z, Zhang $\mathrm{R}$, et al. Can peritumoral radiomics increase the efficiency of the prediction for lymph node metastasis in clinical stage T1 lung adenocarcinoma on CT? Eur Radiol. 2019;29(11): 6049-58.

47 Kuang DM, Peng C, Zhao Q, Wu Y, Chen MS, Zheng L. Activated monocytes in peritumoral stroma of hepatocellular carcinoma promote expansion of memory T helper 17 cells. Hepatology. 2010;51(1):154-64.

48 Chen DP, Ning WR, Li XF, Wei Y, Lao XM, Wang JC, et al. Peritumoral monocytes induce cancer cell autophagy to facilitate the progression of human hepatocellular carcinoma. Autophagy. 2018;14(8):1335-46.

49 Mantovani A, Sozzani S, Locati M, Allavena P, Sica A. Macrophage polarization: tumorassociated macrophages as a paradigm for polarized M2 mononuclear phagocytes. Trends Immunol. 2002;23(11):549-55.

50 Budhu A, Forgues M, Ye QH, Jia HL, He P, Zanetti KA, et al. Prediction of venous metastases, recurrence, and prognosis in hepatocellular carcinoma based on a unique immune response signature of the liver microenvironment. Cancer Cell. 2006;10(2):99-111.
51 Jaffe CC. Measures of response: RECIST, WHO, and new alternatives. J Clin Oncol. 2006;24:3245-51.

52 Aerts HJ, Velazquez ER, Leijenaar RT, Parmar C, Grossmann P, Carvalho S, et al. Decoding tumour phenotype by noninvasive imaging using a quantitative radiomics approach. Nat Commun. 2014;5:4006.

53 Terayama N, Miyayama S, Tatsu H, Yamamoto T, Toya D, Tanaka N, et al. Subsegmental transcatheter arterial embolization for hepatocellular carcinoma in the caudate lobe. J Vasc Interv Radiol. 1998;9(3):501-8.

54 Miyayama S, Matsui O, Taki K, Minami T, Ryu Y, Ito C, et al. Arterial blood supply to the posterior aspect of segment IV of the liver from the caudate branch: demonstration at CT after iodized oil injection. Radiology. 2005;237(3):1110-4

55 Moustafa AS, Abdel Aal AK, Ertel N, Saad N, DuBay D. Saddekni S. Chemoembolization of hepatocellular carcinoma with extrahepatic collateral blood supply: anatomic and technical considerations. Radiographics. 2017;37: 963-77.

56 Boulin M, Adam H, Guiu B, Aho LS, Cercueil JP, Di Martino C, et al. Predictive factors of transarterial chemoembolisation toxicity in unresectable hepatocellular carcinoma. Dig Liver Dis. 2014;46(4):358-62.

57 Lee YK, Kim SU, Kim DY, Ahn SH, Lee KH, Lee DY, et al. Prognostic value of $\alpha$-fetoprotein and des- $\gamma$-carboxy prothrombin responses in patients with hepatocellular carcinoma treated with transarterial chemoembolization. BMC Cancer. 2013;13:5.

58 Tian M, Zhang X, Huang G, Fan W, Li J, Zhang Y. Alpha-fetoprotein assessment for hepatocellular carcinoma after transarterial chemoembolization. NY: Abdom Radiol; 2019.

59 Lencioni R, de Baere T, Soulen MC, Rilling WS, Geschwind JF. Lipiodol transarterial chemoembolization for hepatocellular carcinoma: a systematic review of efficacy and safety data. Hepatology. 2016;64(1):106-16.

60 Trevisani F, Golfieri R. Lipiodol transarterial chemoembolization for hepatocellular carcinoma: Where are we now? Hepatology. 2016; 64(1):23-5

61 Kim DY, Ryu HJ, Choi JY, Park JY, Lee DY, Kim BK, et al. Radiological response predicts survival following transarterial chemoembolisation in patients with unresectable hepatocellular carcinoma. Aliment Pharmacol Ther. 2012;35(11):1343-50. 